\title{
PERFORMANCE AND VALIDATION OF THE 3D ACOUSTIC MODELLING CODE FOR THE EAEG \& SEG 3D MODELLING PROJECT
}

J. BRAC, L. ANNE, A. BAMBERGER and P. DUCLOS

Institut Français du Pétrole, PO Box 311, Rueil-Malmaison Cedex, France

\section{Introduction}

The EAEG\&SEG 3D Modeling Committee decided (Progress Report in Leading Edge and First Break of February, 1994) the main features of the 3D modeling code for the project. A finite difference method was selected in order to model complex structures and a one-parameter acoustic wave equation was accepted. In fact,

- elastic modeling is not essential regarding the problem of complex structural interpretation

- extensive 3D elastic wave propagation computation is still beyond reach of today's

technologies.

The algorithm working group evaluated several finite-difference codes donated to the project and selected the public domain IFP 3D code for its good numerical performances and because it solves practical questions related to input and output data management. The first part of this paper reviews the main features of this code. Three important issues will then be discussed:

- gridding of complex 3D structure by constant parameter grid cell;

- validation of the code and choice of numerical paramaters;

- computational ressources required for the project.

\section{Main features of the code}

The chosen code models $3 \mathrm{D}$ acoustic wave propagation assuming a constant density : the only variable parameter is the acoustic velocity. The acoustic wave equation is discretized by second order explicite scheme in time and high order scheme in space. The order can be set to any value between 4 and 12 .

Efficient absorbing boundary conditions are obtained by the addition of a sponge-like layer to a second order paraxial aproximation of the wave equation. The thickness of the sponge layer is equal to one or two mean wavelengths.

\section{Gridding of the model.}

The problem here is not directly related to the modeling code itself but to the discretization of the model by constant velocity grid cells. As a result, the geometry of the interface between two homogeneous layers would be aproximated by an irregular surface made of steps equal to the discretization mesh size. When the grid interval is about one tenth of the wavelength, this results in diffractions on each step of the discretized interface. They can be seen as a noise disturbing the data. 
An appropriate way of model discretization must be chosen. We propose to use a filter based upon the square of the slowness which rubs out the main part of the spurious diffractions. The space wave lengths of the model and the time wave lengths of the source interact during the propagation process. They must be correctly sampled to ensure a low level of elementary diffraction and dispersion.

\section{Numerical validation of the code.}

This 3D acoustic propagation code has been carefully validated just as much from a cinematic point of view as from a signal amplitude point of view. The validation has been carried out by means of 2 approaches.

First of all, an analytical approach allows the verification of the accuracy of the seismic results. Three kinds of tests depending on the scheme order, the space step, the record duration and the source frequencies, have been done in various media :

- homogeneous media,

- 1D media cylindrically spread in 3D multi-layers media,

- wedge model consisting of an angle includes between 2 straight lines of a given slope.

The first test permits the control of travel times and amplitudes of the direct arrival.

With the multi-layers test, the error on reflectivity can be evaluated.

The diffraction effects on a singularity are tested with the wedge model. Moreover, diffracted waves could also appear along the steps of the dipping interface. Thus, a feedback about the previous choices for gridding the model itself is obtained.

Over and above the tests for layer homogeneous media, a methodology has been defined to test the code in heterogeneous media for which no analytical solution is known.

Let us consider a 2D section in the Overthrust model. A study of the behaviour of the solution when space and time steps become smaller, has stated that the scheme is consistent. Furthermore, solutions with quite large space step solutions converge towards the solution using a very small step.

Similar tests have been carried out in 3D Overthrust sub-models.

\section{Choice of the numerical parameters.}

Taking in reference a $4^{\text {th }}$ order scheme using a small space step, we look for a larger space step tied up with a high order scheme. A comparison of the travel times and the reflectivity on various interfaces leads to choose the following parameters for the Overthrust model:

$$
\begin{aligned}
& \text { - space step }: 25 \mathrm{~m} \text {, } \\
& \text { - time step : } 1 \mathrm{~ms} \text {, } \\
& \text { - } 10^{\text {th }} \text { order scheme in space, } 2^{\text {nd }} \text { order scheme in time, } \\
& \text { - central frequency of the source Ricker }: 15 \mathrm{~Hz} \text {. }
\end{aligned}
$$

Let us consider a $10 \times 10 \times 4 \mathrm{~km}$ domain size and a seismogram recording duration of three seconds. The application of this parameter set leads to the following performance on a one Gigaflops sustained vector supercomputer :

\footnotetext{
- 330 Mbytes memory size,

- 70 minutes per 3D shot point.
} 